\title{
Clinical characteristics and coronary features of coronary ectasia and aneurysm in China*
}

\author{
Zhongwei Cheng $^{\#}$, Yingxian Liü, Shuyang Zhang ${ }^{\dagger}$, Wei Wu, Zhujun Shen, Zhongjie Fan, Hongzhi Xie, \\ Zhenyu Liu, Xiaofeng Jin, Chonghui Wang, Yong Zeng, Quan Fang
}

Department of Cardiology, Peking Union Medical College Hospital, Peking Union Medical College and Chinese Academy of Medical Sciences, Beijing, China

Email: ${ }^{\dagger}$ shuyangzhang103@yahoo.com.cn

Received 29 September 2012; revised 5 November 2012; accepted 25 November 2012

\begin{abstract}
Objective: To describe the clinical characteristics and coronary features of patients with coronary ectasia and aneurysm (CEA) in China. Methods: This was a single center, retrospective clinical study. Patients with CEA who diagnosed by coronary artery angiography (CAG) from April 2003 to October 2009, were enrolled. All patients were followed up by the call or clinic visit. The primary outcome was all-cause mortality or cardiovascular readmission. Results: A total of 39 patients ( 27 males, mean age $59.5 \pm 12.6$ years) diagnosed with CEA, with a prevalence of $0.6 \%$ (a total of 6130 patients underwent CAG during the same period). Thirty-six patients constituted the study population, due to 3 patients lost of followup. The median follow-up was 27 months $(1-76)$. The single vessel was involved in 11 patients (31\%), two vessels were involved in 14 patients $(38 \%)$ and three or more vessels were involved in 11 patients $(31 \%)$. The most common involved vessel was left anterior descending branch $(n=27)$, followed by right coronary artery $(n=23)$, left circumflex branch $(n=20)$ and left main branch $(\mathrm{n}=9)$ ). There was no death during the follow-up. The primary outcome was observed in 8 (22\%) patients. The use of clopidogrel and statins were associated with lower rate of cardiovascular readmission. Conclusion The incidence of CEA was low among patients who referred for CAG in China. The majority of patients had the involvement of multiple vessels, and left anterior descending branch was the most common involved vessel. The CEA patients may benefit from clopidogrel and statins.
\end{abstract}

Keywords: Coronary Ectasia; Coronary Aneurysm;

\footnotetext{
*This study was supported by Grant 30470726 from the Natural Science Foundation of China (to Dr. Shuyang Zhang).

\#The first two authors contributed equally to the work.

${ }^{\dagger}$ Corresponding author.
}

Coronary Feature

\section{INTRODUCTION}

Coronary artery ectasia and aneurysm (CEA) was considered as variants of atherosclerosis. The coronary artery angiography (CAG) showed slow flow of coronary arteries, with the increasing risk of thrombosis. The prevalence of CEA was varied in different countries. The treatment and the efficacy of different medicine were controversial in previous studies [1-5]. However, there was limited data about clinical characteristics and coronary features of CEA patients in China. The aim of the present study was to describe the clinical characteristics and coronary features of CEA patients in China, as well as to evaluate the efficacy of different medicines.

\section{METHODS}

\subsection{Patient Population}

Patients diagnosed with CEA according to CAG in Peking Union Medical College Hospital (PUMCH) from April 2003 to October 2009, were enrolled.

\subsection{Definitions}

Coronary artery ectasia: $>1.5$ times of the diameter of adjacent normal vessels. Coronary artery aneurysm: $>2$ times of the diameter of adjacent normal vessel [1]. Two experienced investigators reviewed the patients' $\mathrm{CAG}$ films and made the diagnosis.

\subsection{Classification of CEA}

The classification of CEA was according to Markis criteria, 4 types were made.

Type 1: Diffuse ectasia of two or three vessels;

Type 2: Diffuse ectasia in one vessel and localized in another;

Type 3: Diffuse ectasia of a single vessel; 
Type 4: Localized or segmental ectasia.

\subsection{Data Collection}

Patient variables including gender, age, blood pressure, history of coronary artery disease, smoking history, hyperlipidemia, diabetes mellitus, body mass index, clinical presentation, coronary angiography results and medications at discharge, were collected.

\subsection{Primary Outcome}

The primary outcome was all-cause mortality or cardiovascular readmission.

\subsection{Statistical Analysis}

All data were analyzed by SPSS software, version 17.0 for Windows, using spearman's correlation analysis to explore the relationship of various medications and the primary outcome. $p$ value $\leq 0.05$ was considered statistically significant.

\section{RESULTS}

\subsection{Patients and Prevalence}

A total of 39 patients ( $69 \%$ male, mean age: $59.5 \pm 12.6$ years) with CEA were diagnosed, 3 patients were lost of follow-up, therefore, 36 patients constituted the study population, including coronary artery ectasia in 32 patients and coronary artery aneurysm in 4 patients. A total of 6130 patients underwent CAG in PUMCH during the same period, therefore, the prevalence of CEA in the patients referred for CAG was $0.6 \%$. All the patients with CEA suffered from angina pectoris, and 10 patients accompanied with atherosclerotic stenosis, the remaining 26 patients $(72 \%)$ were isolated CEA. The median follow-up was 27 months $(1-76)$. Table 1 summarized the clinical characteristics of the study patients.

\subsection{Coronary Features}

The majority of CEA patients were presented with multiple coronary ectasia or aneurysm, the left anterior descending branch (LAD) was the most commonly involved vessel. According to the classification made by Markis, type 1, 2, 3 and 4 were seen in 13, 12, 2 and 9 patients, respectively. Table 2 summarized the coronary features of the study patients.

\subsection{Primary Outcome}

No death was observed. Cardiovascular readmission occurred in 8 patients, including acute myocardial infarction in 3 patients, unstable angina pectoris in 3 patients, acute heart failure in 1 patient and atrial fibrillation in 1 patient, respectively.
Table 1. Clinical characteristics.

\begin{tabular}{cc}
\hline Variable & Patients $(\mathrm{n}=36)$ \\
\hline Age (years, mean $\pm \mathrm{SD})$ & $59 \pm 12$ \\
Body Mass Index $\left(\mathrm{kg} / \mathrm{m}^{2}\right.$, mean $\left.\pm \mathrm{SD}\right)$ & $27 \pm 3$ \\
Male & $26(72 \%)$ \\
Diabetes mellitus & $10(28 \%)$ \\
Hypertension & $23(64 \%)$ \\
Smoking & $17(47 \%)$ \\
Hyperlipidemia & $21(58 \%)$ \\
Family history of coronary artery disease & $8(22 \%)$ \\
Atherosclerotic stenosis & $10(28 \%)$ \\
\hline
\end{tabular}

Table 2. Coronary features.

\begin{tabular}{cc}
\hline \multicolumn{2}{c}{ CAG features of CEA Patients $(\mathrm{n}=36)$} \\
\hline Multi-vessel involvement $(\mathrm{n}>1)$ & $25(70 \%)$ \\
$\mathrm{n}=2$ & $14(39 \%)$ \\
$\mathrm{n}=3$ & $5(14 \%)$ \\
$\mathrm{n}=4$ & $6(17 \%)$ \\
LAD & Coronary distribution \\
RCA & $27(75 \%)$ \\
LCX & $23(65 \%)$ \\
LM & $20(56 \%)$ \\
& $9(25 \%)$ \\
Type1 & \\
Type 2 & $13(36 \%)$ \\
Type 3 & $12(33 \%)$ \\
Type 4 & $2(6 \%)$ \\
\hline
\end{tabular}

$\mathrm{LAD}=$ left anterior descending branch; RCA = right coronary artery; LCX $=$ left circumflex branch; $\mathrm{LM}=$ left main branch.

\subsection{Relationship between Primary Outcomes and Medications}

There was negative correlation between cardiovascular readmission and the current use of clopidogrel $(\mathrm{R}=$ $-0.316, p=0.050)$ and statins $(\mathrm{R}=-0.371, \mathrm{p}=0.026)$. The negative correlation between cardiovascular readmission and the current use of clopidogrel and statins still remained after adjustment of the distribution and classification of CEA. Table 3 summarized the relationship between medications and primary outcome.

\section{DISCUSSION}

Several studies reported the morbidity and cardiovascular risk factors of CEA [2-6]. Coronary Artery Surgery Study reported the largest cohort of CEA patients with a 
Table 3. Correlations between medications and primary outcome.

\begin{tabular}{ccc}
\hline \multirow{2}{*}{ Variable } & \multicolumn{2}{c}{ Primary outcome $(\mathrm{n}=8)$} \\
\cline { 2 - 3 } & $\mathrm{R}$ & $\mathrm{p}$ \\
\hline Aspirin & -0.265 & 0.118 \\
Clopidogrel & -0.316 & 0.050 \\
ARB & -0.263 & 0.122 \\
ACEI & -0.015 & 0.930 \\
Statins & -0.371 & 0.026 \\
Nitrates & 0.104 & 0.546 \\
CCB & -0.215 & 0.209 \\
$\beta$-receptor blockers & -0.226 & 0.185 \\
\hline
\end{tabular}

$\mathrm{ARB}=$ angiotensin 1 receptor blockers; $\mathrm{ACEI}=$ Angiotensin-Converting Enzyme Inhibitors; $\mathrm{CCB}=$ calcium channel blockers.

incidence of $4.9 \%$ among 20,087 patients referred for CAG [2]. In Asian, database containing the largest number of CEA patients is from Singapore, which reporting an incidence of $1.2 \%(n=8641)$ [3]. Our study showed a lower prevalence $(0.6 \%)$ of CEA, which may be attributed to a lower atherosclerotic risk in Chinese population.

All of CEA patients in the present study complained of clinical presentations of angina pectoris, with a male dominance $(69 \%)$, similar with previous reports $[3,5]$. Although atherosclerosis [6], dyslipidemia [5], smoking [5], diabetes mellitus [5], hypertension [7] and abodominal artery aneurysm $[5,8]$ were considered as possible CEA risk factors, there were no significant correlation between these variables and the severity of CEA in the present study.

There were some striking features observed in the present study. The previous study showed that $50 \%$ of patients with CEA also have concomitant atherosclerosis [9], with a most frequently involved branches of RCA, followed by LAD and LCX $[2,4]$, while ectasia of multivessels and LM were rare [9]. However, CEA with atherosclerosis only occurred in $28 \%$ of the present study, while isolated CEA were observed in more than $70 \%$ patients, strongly supporting a recent viewpoint that CEA was not a simple variant of atherosclerosis [10]. Moreover, LAD is the most commonly involved artery, multiple coronaries and LM involvement observed in about $2 / 3$ and $1 / 4$ patients in the present study, respectively.

The appropriate therapeutic strategy is still controversial. Considering that thrombus formation and distal embolization play crucial roles in pathogenesis of CEA [5,11-13], anticoagulation and antiplatelet therapy should be beneficial to reduce cardiovascular events. In case series of Rath [14], 5 patients without obstructive coronary aneurysms developed acute myocardial infarction and complete occlusion of aneurysmal vessels without any anticoagulation and antiplatelet medications. Alloul [15] reported a case with isolated coronary aneurysm, who developed acute myocardial infarction when withdrawal of warfarin after 6 months of preventive anticoagulation. Although many researchers suggested application of platelet inhibitors in all forms of CEA to prevent myocardial ischemia [11,16], long-term follow-up data was limited about the impact of antiplatelet therapy on clinical outcomes of CEAs. Meanwhile, clinical study of anticoagulant medications on CEAs is lack. Our results revealed a significant correlation between clinical improvement and application of aspirin and clopidogrel. In particular, the clopidogrel, which is a strong platelet ADP receptor antagonist, may not only decrease attacks of angina pectoris, but also reduce the readmission event rate. Otherwise, we couldn't find any association between anticoagulation drugs and reduced risks of cardiovascular events, indicating that prophylactic use of low-molecular-weight heparin or warfarin may be not necessary in patients with CEAs.

Statins, which are generally accepted as lipid-lowing and anti-inflammatory agents to protect AS patients from coronary ischemic events, are negative correlation with primary outcomes in the present study. Tokgozoglu et al. [17] reported elevated plasma levels of inflammatory markers such as interleukin-6 and C-reactive protein (CRP) in CEA patients comparing with those with normal coronary. In another study from Ozbay [18], significantly elevated hs-CRP levels in CEA patients than that in AS patients, and significant decreased hs-CRP levels after a 3-month statins therapy, also showing CEA patients may benefit from statins. Aronow [19] and Saratzis et al. [20] reported that patients with abdominal aortic aneurysm could benefit from statins.

\section{Conclusion}

There is a relatively low incidence and overall well outcome of CEA patients in Chinese population. According to the present study, the majority CEA patients were male, with multiple vessels involvement and LAD was the most commonly involved vessel. CEA patients may benefit from clopidogrel and statins. However, the number of the present study is small, further studies are needed to evaluate the effect of different medications on CEA.

\section{REFERENCES}

[1] Hartnell, G.G., Parnell, B.M. and Pridie, R.B. (1985) Coronary artery ectasia. Its prevalence and clinical significance in 4993 patients. British Heart Journal, 54, 392-395. doi:10.1136/hrt.54.4.392

[2] Sway, P.S., Fisher, L.D., Litwin, P., Vignola, P.A., Judkins, M.P., Kemp, H.G., Mudd, J.G. and Gosselin, A.J. 
(1983) Aneurysmal coronary artery disease. Circulation, 67, 134-138. doi:10.1161/01.CIR.67.1.134

[3] Lam, C.S. and Ho, K.T. (2004) Coronary artery ectasia: A ten-year experience in a tertiary hospital in Singapore. Annals, Academy of Medicine, Singapore, 33, 419-422.

[4] Demopoulos, V.P., Olympios, C.D., Fakiolas, C.N., Pissimissis, E.G., Economides, N.M., Adamopoulou, E., Foussas, S.G. and Cokkinos, D.V. (1997) The natural history of aneurysmal coronary artery disease. Heart, 78, 136141.

[5] Baman, T.S., Cole, J.H., Devireddy, C.M. and Sperling, L.S. (2004) Risk factors and outcomes in patients with coronary artery aneurysms. American Journal of Cardiology, 93, 1549-1551. doi:10.1016/j.amjcard.2004.03.011

[6] Maehara, A., Mintz, G.S., Ahmed, J.M., et al. (2001) An intravascular ultrasound classification of angiographic coronary artery aneurysms. American Journal of Cardiology, 88, 365-370. doi:10.1016/S0002-9149(01)01680-0

[7] Markis, J.E., Joffe, C.D., Cohn, P.F., Feen, D.J., Herman, M.V. and Gorlin, R. (1976) Clinical significance of coronary arterial ectasia. American Journal of Cardiology, 37, 217-222. doi:10.1016/0002-9149(76)90315-5

[8] Mariscalco, G., Mantovani, V., Ferrarese, S., Leva, C., Orru, A. and Sala, A. (2006) Coronary artery aneurysm: Management and association with abdominal aortic aneurysm. Cardiovascular Pathology, 15, 100-104. doi:10.1016/j.carpath.2005.11.005

[9] Cohen, P. and O'Gara, P.T. (2008) Coronary artery aneurysms: A review of the natural history, pathophysiology, and management. Cardiology in Review, 16, 301304. doi:10.1097/CRD.0b013e3181852659

[10] Yetkin, E. and Waltenberger, J. (2007) Novel insights into an old controversy: Is coronary artery ectasia a variant of coronary atherosclerosis? Clinical Research in Cardiology, 96, 331-339. doi:10.1007/s00392-007-0521-0

[11] Krüger, D., Stierle, U., Herrmann, G., Simon, R. and Sheikhzadeh, A. (1999) Exercise-induced myocardial ischemia in isolated coronary artery ectasias and aneurysms ("dilated coronopathy"). Journal of the American College of Cardiology, 34, 1461-1470. doi:10.1016/S0735-1097(99)00375-7

[12] Akyurek, O., Berkalp, B., Sayin, T., Kumbasar, D., Ker- vancioğlu, C. and Oral, D. (2003) Altered coronary flow properties in diffuse coronary artery ectasia. American Heart Journal, 145, 66-72. doi:10.1067/mhj.2003.48

[13] Aboud, A., Bossert. T., Sigusch, H.H. and Gummert, J. (2007) Surgical treatment of multiple atherosclerotic coronary artery aneurysms in a patient with acute myocardial infarction. Clinical Research in Cardiology, 96, 45-48. doi:10.1007/s00392-006-0453-0

[14] Rath, S., Har-Zahav, Y., Battler, A., Agranat, O., Rotstein, Z., Rabinowitz, B. and Neufeld, H.N. (1985) Fate of nonobstructive aneurysmatic coronary artery disease: Angiographic and clinical follow-up report. American Heart Journal, 109, 785-791. doi:10.1016/0002-8703(85)90639-8

[15] Alloul, S., Dahdah, N. and Miró, J. (2009) Thrombus in a coronary artery aneurysm shortly after warfarin withdrawal. Pediatric Cardiology, 30, 188-190. doi:10.1007/s00246-008-9290-4

[16] Turhan, H. and Yetkin, E. (2007) What is the plausible strategy for the management of patients with isolated coronary artery ectasia and myocardial ischemia? International Journal of Cardiology, 117, 285-286. doi:10.1016/j.ijcard.2006.05.042

[17] Tokgozoglu, L., Ergene, O., Kinay, O., Nazli, C., Hascelik, G. and Hoscan, Y. (2004) Plasma interleukin-6 levels are increased in coronary artery ectasia. Acta Cardiologica, 59, 515-519. doi:10.2143/AC.59.5.2005226

[18] Ozbay, Y., Akbulut, M., Balin, M., Kayancicek, H., Baydas, A. and Korkmaz, H. (2007) The level of hs-CRP in coronary artery ectasia and its response to statin and angiotensin-converting enzyme inhibitor treatment. Mediators of Inflammation, 2007, 89649. doi:10.1155/2007/89649

[19] Aronow, W.S. (2011) Peripheral arterial disease and abdominal aortic aneurysm in elderly people. Minerva Medica, 102, 483-500.

[20] Saratzis, A., Saratzis, N., Melas, N. and Kiskinis, D. (2008) Pharmacotherapy before and after endovascular repair of abdominal aortic aneurysms. Current Vascular Pharmacology, 6, 240-249. doi:10.2174/157016108785909689 